\title{
Age affects pigeon memory capacity, but not representation of serial order, during a locomotor sequential-learning task
}

\section{Christina Meier}

University of Manitoba

\section{Parisa Sepehri}

University of Manitoba

Debbie M. Kelly ( $\sim$ Debbie.Kelly@umanitoba.ca )

University of Manitoba

\section{Research Article}

Keywords: Aging, locomotor, memory, pigeons, sequential learning, serial order, simultaneous chaining

Posted Date: March 15th, 2021

DOI: https://doi.org/10.21203/rs.3.rs-289410/v1

License: (c) (i) This work is licensed under a Creative Commons Attribution 4.0 International License.

Read Full License 
1 Age affects pigeon memory capacity, but not representation of serial order, during a

2

3

4

5

6

7

8

9

10

$11 *$ Corresponding author:

12 Debbie M. Kelly

13 Department of Psychology

14 P438 Duff Roblin Bldg., 190 Dysart Rd.

15 University of Manitoba, Winnipeg, MB, R3T 2N2 Canada

16 Email: Debbie.Kelly@umanitoba.ca

17 Telephone: +1 2044749489

18

19

20

21

22 Manuscript being prepared for publication. Please do not cite or quote without permission

23 from an author.

24 (C) Copyright 2021, the authors 

order, during a locomotor sequential-learning task

\section{ABSTRACT}

Aging affects individuals of every species, with sometimes detrimental effects on

30 memory and cognition. The simultaneous-chaining task, a sequential-learning task, requires

31 subjects to select items in a predetermined sequence, putting demands on memory and

32 cognitive processing capacity. It is thus a useful tool to investigate age-related differences in

33 these domains. Pigeons of three age groups (young, adult and aged) completed a locomotor

34 adaptation of the task, learning a list of four items. Training began presenting only the first

35 item; additional items were added, one at a time, once previous items were reliably selected

36 in their correct order. Although memory capacity declined noticeably with age, not all aged

37 pigeons showed impairments compared to younger pigeons, suggesting that inter-individual

38 variability emerged with age. During a subsequent free-recall memory test, when all trained

39 items were presented alongside novel distractor items, most pigeons did not reproduce the

40 trained sequence in the absence of reinforcement. During a further forced-choice test, when

41 pigeons were given a choice between only two of the trained items, all three age groups

42 showed evidence of an understanding of the ordinal relationship between items by choosing

43 the earlier item, indicating that complex cognitive processing, unlike memory capacity,

44 remained unaffected by age.

45

46 KEYWORDS

47 Aging, locomotor, memory, pigeons, sequential learning, serial order, simultaneous chaining. 
As we age, so does our brain, and with it many cognitive functions decline. Age-related

49 degeneration of cognitive and memory capacities is well-documented for humans, and is

50 evident even for healthy individuals ${ }^{1,2}$. The impact of aging on nonhuman animal cognition

51 is less well explored ${ }^{3}$, but this knowledge is important for understanding common effects of

52 non-pathological aging on cognition. The aging process affects every living being regardless

53 of species, but the way in which it manifests in cognitive changes or impairments can

54 sometimes differ greatly among individuals. Establishing how, and to what degree, aging can

55 affect vital cognitive capacities is a first step in assessing the range of normal and abnormal

56 impairments.

57 The simultaneous-chaining task ${ }^{4,5}$ is cognitively demanding as it requires subjects to

58 reproduce a list of items in a specific sequence, with the only feedback provided regarding

59 the correctness of a choice being the continuation of the trial. This task puts demands on an

60 individual's memory capacity, in terms of both reference memory to learn the sequence, and

61 working memory to update the last choice made in order to determine the next required

62 response. An individual's memory capacity can be measured through the successive chaining

63 of subsequent sequence items ${ }^{6}$. Each time a subject learns to respond correctly to a sequence

64 of $n$ items, another item is added to the chain $(n+1)$. Using this method, pigeons have

65 successfully been trained to reproduce lists of four to five items ${ }^{4,5}$.

66 In addition to the task's adoption for evaluating memory capacity, it has also been

67 selected as an assessment tool for cognitive capacity, and as such has been used to study a

68 diverse range of species, from humans and apes to pigeons and chickens ${ }^{4}$. As suggested by

69 the task name, successful acquisition of the sequence can be achieved through simple

70 associative chaining, with a response to the first item serving as a cue to respond to the

71 second item, and so on. However, a more cognitively complex solution would be to form a

72 mental representation of the item order, whereby each item in the sequence is acquired not 
73 only relative to the immediately preceding and following items, but also in terms of its unique

74 ordinal position within the sequence as a whole (e.g., is the item the first, second, third, etc.).

75 One way to assess a subject's ability to form a representation of order is during subsequent

76 pairwise-choice tests, for which only two items of the learned sequence are presented

77 together, and the subject is allowed a single choice. If item order had been represented,

78 subjects would be expected to recognise the ordinal relationship between the two presented

79 items even in the absence of the complete sequence, and accordingly to choose the item that

80 appeared earlier in the training sequence. However, if learning occurred based on associative

81 chaining alone, subjects would be expected to perform well only when the first item is

82 present, but to be unable to distinguish between any later items, as the cue to respond to

83 either - a successful response to the preceding item - would not have occurred.

84 During Terrace's ${ }^{5}$ studies, pigeons largely responded in line with the predictions for

85 an associative-chaining account, in that for trials in which one of the presented items was the

86 first item of the trained sequence, that item was chosen correctly, and for trials in which the

87 first item was not part of the choice, a choice was made at chance. However, Terrace also

88 found one notable exception to this pattern: when a choice included the last sequence item,

89 the other item was reliably chosen, and the last item was avoided. Terrace 5 interpreted these

90 results by assuming that pigeons had learned the special status of the two items to which a

91 response was required first and last, but that they otherwise did not possess a mental

92 representation of the ordinal relationship between two list items. More recently, however,

93 Scarf and Colombo ${ }^{4}$ argued that pigeons are indeed able to form a mental representation of

94 the sequence order. Proposing that their previous inability to maintain the trained order

95 during two-item tests was due to a contextual change resulting from presenting only two

96 items rather than the full sequence, Scarf and Colombo suggested that this change may have

97 disrupted the pigeons' ability to relate the test to the training and transfer the task 
98 requirements accordingly. Taking this critique into consideration, in our current study we

99 presented subjects with a free-recall test, as well as the conventional pairwise-choice test.

100 During the free-recall memory test, all items of the trained sequence were presented, thus

101 preventing the sudden perceptual change that arguably impeded a transfer from training to

102 test. In addition, previously unseen distractor items were shown alongside the familiar

103 training items. Instead of just one choice, subjects were free to make several choices, in order

104 to measure both item recognition and adherence to the trained order.

105 In summary, the simultaneous-chaining task provides an excellent procedure for

106 investigating age-related effects of memory decline, and for comparing cognitive abilities

107 across species. The goals of our study were two-fold. Firstly, to investigate potential age-

108 related decline in memory capacity and secondly, to elucidate the underlying cognitive

109 mechanisms governing task acquisition for pigeons. To achieve the former, we assessed the

110 length of a sequence that a subject could acquire within a reasonable duration of training. For

111 the latter, we investigated whether a subject's age influenced its' ability to create a mental

112 representation of the trained order.

113

\section{METHODS}

\section{Subjects}

116 Twelve locally sourced racing pigeons (Columba livia) completed this experiment.

117 They were grouped into three categories according to their age at test: young (1 year of age;

$118 N=4,2$ females), adult (6-8 years of age; $N=4,2$ females) and aged (15-17 years of age; $N=$

1194,1 female). The pigeons were housed in individual metal (60 x $60 \times 38 \mathrm{~cm}$ length $\mathrm{x}$ width $\mathrm{x}$

120 height $)$ or plastic $(75 \times 70 \times 50 \mathrm{~cm})$ cages in a colony room at the Department of Psychology

121 of the University of Manitoba. The room was kept year-round at a temperature of $21^{\circ} \mathrm{C}$ and a

122 12-hour light-dark cycle with lights on at 0700 hours. The pigeons' weights were monitored 
123 daily and maintained, through controlled feeding of a mixture of yellow and green peas, oat

124 groats, red milo, yellow popping corn, and white millet, at $90 \%$ of their free-feeding weight

125 and had ad lib access to water and grit inside their cage. The adult and aged pigeons had

126 previous testing experience, but all were naïve to the specific procedures of this experiment.

\section{Ethical Note}

128 This study was approved by the University of Manitoba's Local Animal Use Committee

129 (protocol number F18-042) in accordance with the Canadian Council on Animal Care and the 130 ARRIVE guidelines.

\section{$131 \quad$ Apparatus}

132 Training and testing sessions were conducted in an enclosed rectangular arena made 133 of Styrofoam ${ }^{\circledR}$. The walls $\left(60 \mathrm{~cm}\right.$ tall) were lined with white Con-Tact ${ }^{\circledR}$ paper to permit 134 cleaning. The base (200 x $100 \mathrm{~cm}$ length $\mathrm{x}$ width) was covered with black rubber flooring. 135 White shower curtains enclosed the arena to block access to external visual cues. Six feeders 136 were placed in a circular array inside the arena and fixed to the floor with Velcro (see Figure 137 1). Each feeder consisted of a wooden base (10 x $10 \times 10 \mathrm{~cm}$ length $\times$ width $\mathrm{x}$ height $)$ and a 138 wooden ramp covered with sandpaper $\left(10 \times 20 \mathrm{~cm}\right.$ base with a $26.6^{\circ}$ incline $)$ leading up to 139 the base. A plastic cup (6 cm diameter) was attached to the inside of the base. To make the 140 inside of the feeder only visible from the top of the ramp, the three sides of the base facing 141 away from the ramp were lined with white paper (30 cm height), and a white foam cone was 142 attached to the top of the paper barrier. The white cones could be replaced by equally-sized 143 cones of different colours to make each feeder visually distinct (herein these coloured cones 144 are referred to as features). A centrally-mounted Logitech HD Webcam C270, connected to a 145 Dell desktop computer (running 64-bit Windows 10 Enterprise) in the adjacent room, was 146 suspended from the ceiling for recording of trials. Two Conair ${ }^{\circledR}$ white noise generators were 147 placed external to the arena, at opposing corners to mask sounds. 


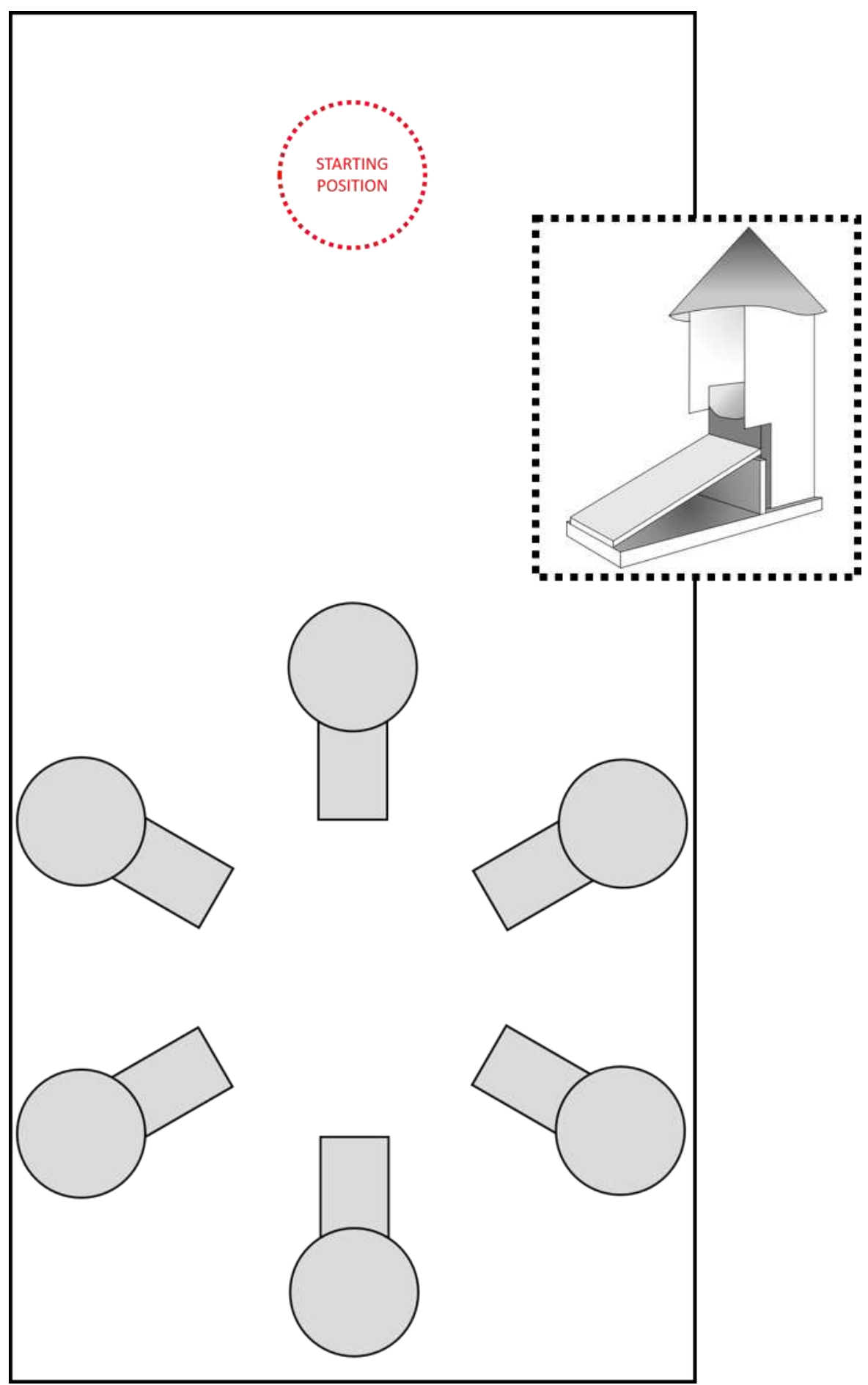

Figure 1. Top-down view of the inside of the experimental arena. The six feeders (in grey) were placed in a circular array in one half of the arena. The pigeon entered at the opposite side of the arena (the approximate location is marked in red). The insert in the broken-line box shows a 3D model of a feeder. 


\section{$151 \quad$ Procedure}

\section{General Procedure}

153 A pigeon was transported from the colony room to the procedure room in an opaque

154 white transport container, which also served as a holding container between trials. At the 155 beginning of each trial, the lights in the procedure room and arena were extinguished, and 156 the pigeon was removed from the holding container and placed into the arena at the starting 157 position (see Figure 1). The experimenter exited the procedure room and remotely 158 illuminated the procedure room lights, which delimitated the start of a trial. Once the pigeon 159 completed the choice requirement (see below), or ten minutes elapsed, whichever occurred 160 first, the lights were extinguished, and the pigeon was removed from the darkened arena and 161 returned to the holding container where it remained while the arena was prepared for the 162 next trial.

163 Feeder Training Phase

164 To train the pigeons to retrieve maple peas from the feeders, a single feeder with a 165 white cone (herein referred to as a white feeder) was located in one of the six possible array 166 locations (with location counterbalanced across trials). Five maple peas were placed at each 167 of the following locations: in the plastic cup inside the feeder, on the ramp, and on the floor 168 of the arena at the base of the ramp. With training, the number of maple peas was gradually 169 reduced from fifteen to two, and only placed inside the feeder. Pigeons were given a 170 maximum of 60 minutes to consume the maple peas. A trial ended 30 seconds after the last 171 maple pea was consumed, after which the arena was re-set and a new trial started. As each 172 daily session lasted a maximum of 60 minutes, a pigeon could receive between one and twelve 173 trials per daily session. Pigeons experienced trials with a single feeder until all maple peas 174 were consumed within 5 minutes of trial start, for a minimum of 12 trials in total. Next, 
175 pigeons were presented with all six identical feeders arranged in the circular array, with one

176 maple pea inside each feeder. Pigeons progressed to the Memory Training phase once every

177 feeder was visited within the first 5 minutes of a trial for 5 consecutive trials.

178 Memory Training Phase

179 A daily training session consisted of 10 trials, with the six feeders positioned in the

180 circular array formation. Across four distinct stages, the pigeons were trained to visit four of

181 the six feeders in a specific sequence, as indicated by distinctly coloured features (red, green,

182 blue, yellow, orange and purple; herein referred to as a sequence item). The order in which

183 the feeders had to be visited was consistent for each pigeon, but pseudo-counterbalanced

184 among the pigeons such that one of the six colours was assigned to be the first item in the

185 sequence for two pigeons. The location of each sequence item within the array was

186 randomised across trials so that only the colours on the feeders, but not the feeder locations

187 within the arena or relative to each other, indicated the correct sequence.

188 Originally, the stages described below were administered with all six coloured

189 features placed on the feeders. However, although all subjects quickly passed the first

190 training stage, during which only the first item in the sequence was reinforced, acquisition of

191 any following memory items appeared hindered by this procedure. In particular, following a

192 successful visit to the first item, the subjects stopped exploring the arena and were reluctant

193 to visit any additional feeders, as visiting them had previously not been reinforced. For the

194 majority of subjects, the transition to training stage 2 resulted in reduced attention to the

195 previously learned first item. Following ten sessions of continued decline in performance by

196 all subjects, we revised the training procedures to encourage exploration of the area after the

197 first item had been visited. Thus, the entire training phase was restarted from stage 1 for all 198 subjects as follows. 
200 presented with five white feeders and the first sequence item. Each successive training stage

201 differed from the previous in that the next new sequence item replaced one of the white

202 feeders in the array. Whereas the white feeders were always non-reinforced, during TS1, four

203 maple peas were placed into the first sequence item; the pigeon was allowed to inspect any

204 feeder. During training stage 2 (TS2; Figure 2B), three maple peas each were placed into the

205 first and second sequence items. If the pigeon approached any feeder before visiting the first

206 sequence item, the lights were extinguished immediately to indicate an incorrect response

207 and the trial ended. If the pigeon successfully visited the first sequence item, it could inspect

208 any other feeder. During training stage 3 (TS3; Figure 2C), two maple peas each were placed

209 into the three sequence items. Only after the pigeon had approached the first and second

210 sequence items, it was allowed to inspect any feeder. During training stage 4 (TS4; Figure

211 2D), two maple peas each were placed into the four presented sequence items. Only after

212 visiting the first, second and third items in sequence, the pigeon was allowed to freely inspect

213 any feeder. During each stage, a trial ended with the extinction of the lights after the pigeon

214 consumed all available peas and exited the final feeder, or if the pigeon visited the feeders in

215 any other order than described, or after ten minutes of inactivity. Training progressed to the

216 next stage once a pigeon consistently visited all presented sequence items in the correct order

217 without visiting any other feeders in at least seven out of ten trials of a session, for two

218 consecutive sessions. If a pigeon failed to advance from one training stage to the next within

21960 sessions, it was considered to have reached the maximum number of sequence items it

220 could memorise, and proceeded to the Testing phase.

221 Testing Phase

222 Two tests were administered in alternating blocks of three sessions: a choice test, 223 consisting of nine sessions, and a memory test, consisting of a minimum of six sessions. The 
224 tests served to examine the successful encoding of the trained sequence items, both regarding

225 their identity (colour) and their position within the sequence. To this effect, the previously

226 trained items were presented alongside novel items of the colours not used during the

227 subject's training.

Choice Test. This test recreated the pairwise forced-choice tests conducted in

229 previous studies on sequence learning to determine whether there was evidence of a mental

230 representation of the trained order. Each session consisted of five reinforced trials, which

231 were identical to training trials during the final stage a pigeon had reached, and five non-

232 reinforced test trials, presented in alternating fashion. During test trials, two feeders had

233 coloured features. All 15 possible combinations of the coloured features were presented once

234 per three-session block, resulting in a total of 45 test trials. Test trials ended after the first

235 visit to any feeder.

236

237

a: Training Stage 1

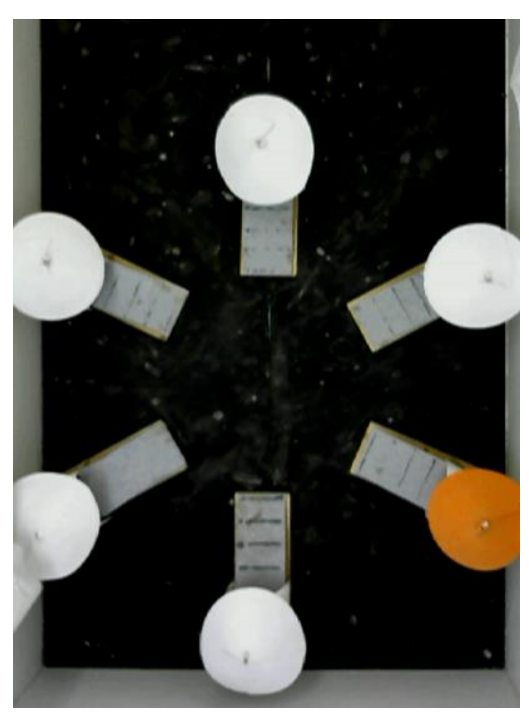

c: Training Stage 3 b: Training Stage 2

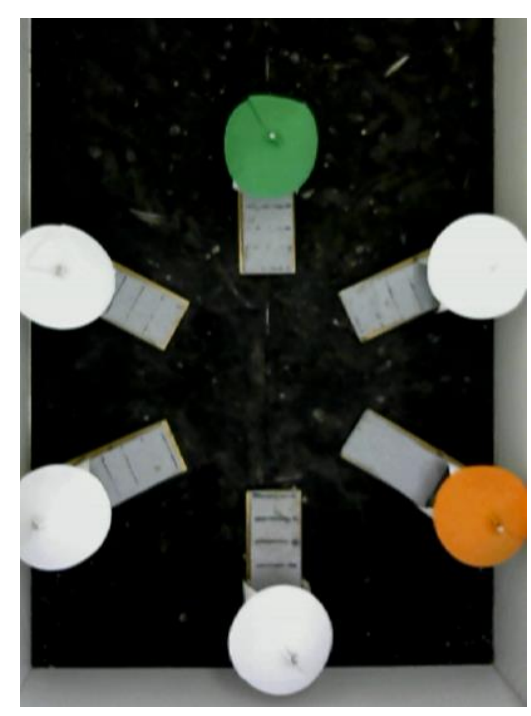

d: Training Stage 4 

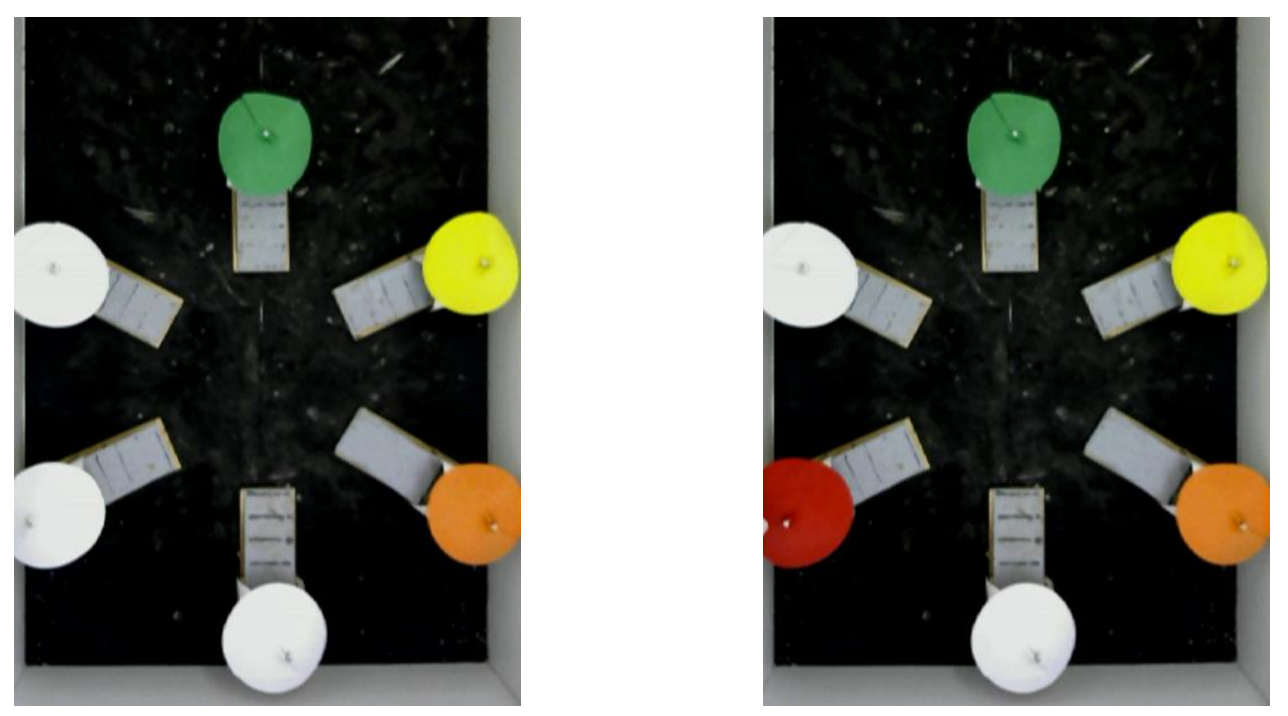

Figure 2. Examples of a trial in each memory training stage. The location of each sequence item (coloured feeder) changed between trials as to not systematically reinforce a certain location. The sequence shown here is for illustrative purposes.

241 disruptive effect of pairwise tests on performance potentially being due to the change in

242 presentation of the sequence items, we additionally incorporated an open memory test

243 presenting all sequence items and novel items together. Each session started with at least two

244 reinforced trials, which were identical to training trials during the final stage a pigeon had

245 reached. Following two correct baseline trials, a non-reinforced test trial was administered,

246 during which all six (sequence and novel) items in a predetermined, randomised order. Test

247 trials ended after the pigeon made four visits, including repeat visits to the same feeder.

248 Memory test sessions were continued until a pigeon had completed ten test trials. Due to the

249 criterion of two correct baseline trials preceding any test trial, each session could contain 250 between zero and three test trials.

\section{RESULTS}

\section{Data Collection}


Data collection was prematurely terminated due to restrictions put in place by the

255 University of Manitoba in response to the Covid-19 pandemic. Two young subjects had

256 completed 30 and 44 sessions of TS4, respectively, and one aged subject had completed 4

257 sessions of TS4 when the experiment was ceased; only their data from the completed TS3

258 were included in the analysis to provide an accurate image of learning. One adult subject

259 (Orange 11) was in the middle of the test phase when data collection was suspended; for this

260 subject, all completed tests sessions were included in the data analysis (providing data for 35

261 of the 45 Choice Test trials and 3 of 10 Memory Test trials).

\section{Data Analysis}

263 Given the format of the training data, we ran a linear mixed model (LMM) with the

264 simple factor Age Group (young, adult, aged) and the polynomial factor Training Stage (TS1,

265 TS2, TS3, TS4), the hatch year of subjects as an unscaled covariate and pigeon identity as the

266 cluster variable assuming correlated effects. Planned pairwise comparisons of performance

267 were conducted as $t$-tests. A simple-effect analysis was conducted to investigate the presence

268 of age-group differences within training phases. Lastly, a Kruskal-Wallis non-parametric one-

269 way ANOVA was performed to assess potential age-group differences in the highest daily

270 performance score achieved during the first half of training in TS4.

271 Analyses of test performance were carried out separately for the two groups of

272 pigeons for which sequence item 3 or 4 had been the last trained item. For the Choice Test,

273 choices between two previously trained items were assessed by conducting Friedman non-

274 parametric repeated-measures ANOVAs on the percentage of trials during which a subject

275 chose the item that had appeared earlier in the sequence, for each combination of trained

276 sequence items. Choices between a trained and a novel item were assessed by conducting

277 Friedman non-parametric repeated-measures ANOVAs on the percentage of trials during

278 which a subject chose a previously trained item over a novel item. To meaningfully analyse 
279 performance in the Memory Test, we considered the correct execution of smaller "chunks"

280 within the trained sequence, specifically, a subject's tendency to choose sequence item 1 first

281 before visiting any other item, to choose at least two sequence items in their correct order

282 (i.e., 1 followed by 2, 2 followed by 3, or 3 followed by 4, regardless of which item was chosen

283 before or after this isolated combination of two items), or to choose the final trained item last.

284 Friedman non-parametric repeated-measures ANOVAs were performance on the percentage

285 of trials during which a subject completed each of these chunks. For any of these ANOVAs,

286 pairwise post-hoc Durbin-Conover comparisons between individual factor levels were

287 performed if an analysis indicated a significant main effect. All analyses were carried out in

288 jamovi version $1.2^{7}$.

289 Memory Training

290 All 12 subjects successfully passed TS1 and TS2, with the exception of one aged 291 subject that failed to continuously search the feeders during TS2 and was removed from the

292 experiment. First performance differences emerged in TS3, as only two subjects each in the 293 adult and aged group reached the training criterion, in contrast to all four subjects in the 294 young group that passed this stage. Finally, none of the subjects that entered TS4 were able 295 to pass the criterion within the number of training sessions they received.

296 The analysis revealed a significant effect of the factor Training Stage (LMM: $F_{3,18.78}=$ 297 130.14, $P<0.001$; Figure 3), as the number of training sessions required to pass a stage 298 increased as more sequence items were added during consecutive stages, assuming a 299 significant cubic function (LMM: $\left.t_{17.72}=5.67, P<0.001\right)$. Pairwise comparisons confirmed 300 that, although training performance in TS1 and TS2 did not differ significantly $\left(t\right.$-test: $t_{19.1}=$ $3012.60, P=0.11$ ), the number of training days required to pass TS3 was significantly higher 302 compared to the former two stages ( $t$-tests: TS3 vs. TS1: $t_{19.1}=15.37, P<0.001$; TS3 vs. TS2: 
$\left.t_{18.4}=12.54, P<0.001\right)$, and higher yet again in TS4 ( $t$-tests: TS4 vs. TS3: $t_{21.2}=2.92, P=0.049$;

304 comparisons to TS1 and TS2: both $P \leq 0.001$ ).

305 The factor Age Group had no relevant influence on the number of training sessions

306 required to pass a training stage across all four stages, as no age group showed a consistently

307 lower or higher number of sessions than the other groups (LMM: $F_{2,6.65}=0.87, P=0.462$ ).

308 However, the emerging interaction effect of the two factors (LMM: $F_{6,18.76}=2.62, P=0.051$ )

309 pointed towards differences between age groups within single training stages, confirmed by

310 the subsequent simple-effect analysis; although subjects within the three age groups were

311 equally successful in TS1 (ANOVA: $F_{2,10.5}=0.03, P=0.97$ ) and TS2 (ANOVA: $F_{2,10.6}=0.18, P=$

3120.84 ), this was not the case for TS3 (ANOVA: $F_{2,10.6}=5.46, P=0.024$ ). For TS3, young subjects

313 performed significantly better than their adult counterparts $\left(t\right.$-test: $t_{9.08}=2.27, P=0.049$; the

314 high variance within the aged group makes comparisons to this age group inconclusive, both

$315 P \geq 0.346)$. In contrast, all three age groups were equally unable in completing TS4 within the

31660 training sessions provided (ANOVA: $F_{2,12.1}=0.03, P=0.97$ ).

317 To obtain a measure of progress for TS4, we evaluated the highest daily performance

318 score (correctly completed trials out of the ten daily trials) obtained halfway through the

319 training stage, after 30 sessions of TS4 had been completed. This allowed us to include the

320 data from the two young subjects that had to cease training before completing all 60 sessions

321 of TS4. The analysis revealed that there were no statistically significant differences between

322 the three age groups in this early performance level (Kruskal-Wallis test: $\chi^{2}{ }_{2}=1.56, P=$ 323 0.459).

324 In summary, increasing the number of sequence items with each training stage led to 325 an increase in the number of sessions required to acquire the sequence. Age differences were 326 only notable for TS3, as young subjects were able to successfully complete this stage within 327 fewer sessions than adult or aged subjects. 


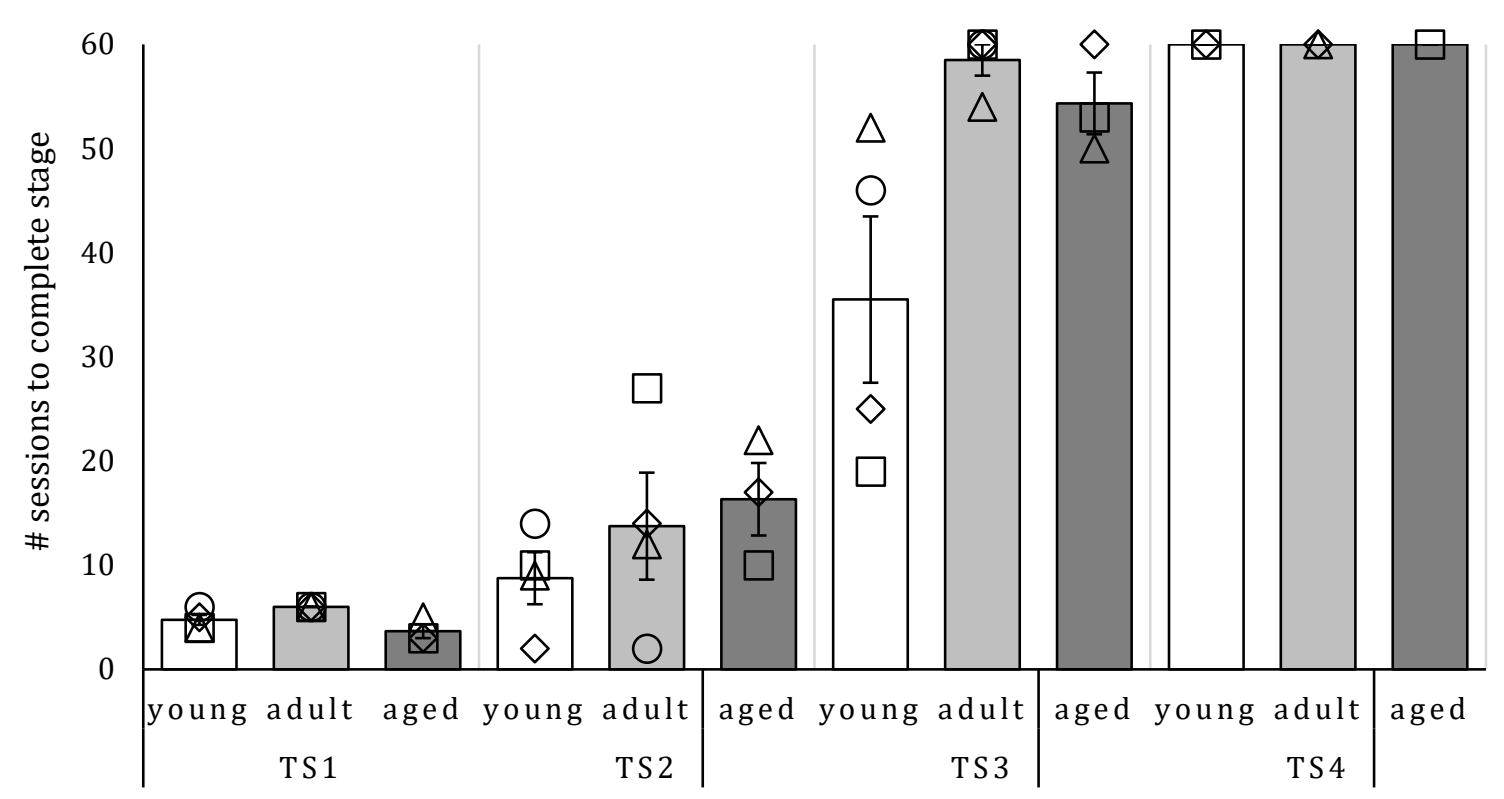

Figure 3. Mean number of sessions required to reach completion criterion in each training stage (TS) for each age group. Individual subject data are plotted as point values. Error bars represent standard errors.

\section{Testing}

332 Of the 12 pigeons that started the experiment, six completed all nine sessions of the

333 Choice Test and sufficient sessions of the Memory Test to accumulate at least ten Memory

334 Test trials. Given the low number of subjects, we analysed individual performance (see Tables

3351 through 3) and grouped subjects based on the number of sequence item they had been

336 trained on instead of age groups. To facilitate the description and analysis of the tests, in

337 addition to the sequence items used during training (i.e., sequence items 1 through 4), the

338 novel items presented during the tests were numbered consecutively (i.e., sequence items 5

339 and 6). However, there was no qualitative difference to the latter two items.

340 Choice Test 
342 studies on sequence learning to determine whether subjects had encoded the trained order.

343 Table 1 shows the percentage of trials during which a subject chose the item that had

344 appeared earlier in the sequence for each combination of trained sequence items. For the

345 subjects experiencing up to TS3, although performance was numerically worse for choices

346 between items 2 and 3, there was no statistically significant difference in performance

347 between choices (Friedman test: $\chi^{2}{ }_{2}=2.6, P=0.273$ ). However, for the four subjects that had

348 reached TS4, whether or not the earlier item was chosen significantly depended on the

349 presented options (Friedman test: $\chi^{2}{ }_{5}=13.5, P=0.019$ ). The pigeons consistently chose item

3501 when it was presented against any other item, and preferred item 2 in choices between

351 items 2 and 4, but were significantly less likely to choose the earlier item for choices between

352 items 3 and 4 and for choices between items 2 and 3, as confirmed by pairwise post-hoc

353 comparisons (Durbin-Conover tests: comparing choices 2 vs. 3 and 3 vs. 4 to all other choices:

354 all $P=0.004$; all other comparisons: all $P=1.0$ ). These results confirm that sequence item 1

355 was reliably recognised as the first item to attend to, whereas choices between other

356 sequence items, including the final item, were less accurate.

Table 1. Percentage of trials (out of three trials) during which a subject chose the item that had appeared earlier in the sequence. Individuals are listed in order of the number of items they received during training; those that only completed up to TS3 were not presented with choices including sequence item 4.

choice between sequence items

\begin{tabular}{lrrrrrr} 
Subject & 1 vs 2 & 1 vs 3 & 1 vs 4 & 2 vs 3 & 2 vs 4 & 3 vs 4 \\
\hline Yellow 23 & $100 \%$ & $67 \%$ & - & $100 \%$ & - & - \\
Green 1076 & $100 \%$ & $67 \%$ & - & $0 \%$ & - & - \\
DV 312 & $100 \%$ & $100 \%$ & - & $33 \%$ & - & - \\
\hline Average & $\mathbf{1 0 0 \%}$ & $\mathbf{7 8 \%}$ & - & $\mathbf{4 4 \%}$ & - & - \\
\hline \hline Yellow 2 & $100 \%$ & $100 \%$ & $100 \%$ & $33 \%$ & $100 \%$ & $67 \%$
\end{tabular}




\begin{tabular}{lrrrrrr} 
White 8174 & $100 \%$ & $100 \%$ & $100 \%$ & $0 \%$ & $100 \%$ & $100 \%$ \\
Orange 11 & $100 \% *$ & $100 \%$ & $100 \% *$ & $50 \%^{*}$ & $100 \%$ & $0 \%$ \\
DV 573 & $100 \%$ & $100 \%$ & $100 \%$ & $100 \%$ & $100 \%$ & $67 \%$ \\
\hline Average & $\mathbf{1 0 0 \%}$ & $\mathbf{1 0 0 \%}$ & $\mathbf{1 0 0 \%}$ & $\mathbf{4 6 \%}$ & $\mathbf{1 0 0 \%}$ & $\mathbf{5 8 \%}$ \\
\hline
\end{tabular}

* - based on two trials instead of three.

In addition to the above, we examined pigeons' overall encoding of the identity of

362 trained sequence items by examining their choices when presented with a trained sequence

363 item and a novel item. Table 2 shows the percentage of trials during which a subject chose

364 the trained item for such choices. For those subjects that only completed TS3, the percentage

365 of choice for the known item did not significantly change depending on which trained

366 sequence item was presented (Friedman test: $\chi^{2}{ }_{2}=1.64, P=0.44$ ). For subjects experiencing

367 TS4, the percentage of choice for the known item also did not significantly change with the

368 presented items (Friedman test: $\chi^{2}{ }_{3}=6.26, P=0.099$ ), although the pigeons tended to choose

369 trained items that appeared earlier in the sequence. Pairwise post-hoc comparisons (Durbin-

370 Conover) confirmed that the choice of item 1 was significantly higher than choices of items 3

371 or 4 (comparisons of "known option was item 1 " to either other option: both $P \leq 0.029$;

372 comparison of "known option was item 1" to "known option was item 2": $P=0.071$; all other

373 comparisons: $P \geq 0.48$ ). This result indicates that, when trained with four items, the

374 preference for a known item over a novel item decreased the later the item appeared in the

375 trained sequence.

Table 2. Percentage of Choice Test trials during which a subject chose the known item when the choice was between a known (sequence items 1, 2, 3, or 4, as applicable) and an unknown item (sequence items 5, 6, or 4, as applicable), or between two unknown items. Individuals are listed in order of the number of items they were trained on; those that only completed up to TS3 were not presented with sequence item 4 as a known option. 


\begin{tabular}{lrcrr}
\hline & \multicolumn{3}{c}{ known option was } \\
Subject & Item 1 & Item 2 & Item 3 & Item 4 \\
\hline Yellow 23 & $100 \%$ & $67 \%$ & $70 \%^{\$}$ & - \\
Green 1076 & $89 \%$ & $89 \%$ & $100 \%$ & - \\
DV 312 & $89 \%$ & $56 \%$ & $50 \%^{\$}$ & - \\
\hline Average & $\mathbf{9 3 \%}$ & $\mathbf{7 1 \%}$ & $\mathbf{7 4 \%}$ & - \\
\hline Yellow 2 & $100 \%$ & $100 \%$ & $67 \%$ & $100 \%$ \\
White 8174 & $100 \%$ & $83 \%$ & $83 \%$ & $67 \%$ \\
Orange 11 & $80 \% *$ & $75 \%{ }^{\wedge}$ & $60 \%{ }^{*}$ & $25 \%^{\wedge}$ \\
DV 573 & $100 \%$ & $50 \%$ & $67 \%$ & $67 \%$ \\
\hline Average & $\mathbf{9 5 \%}$ & $\mathbf{7 7 \%}$ & $\mathbf{6 9 \%}$ & $\mathbf{6 5 \%}$ \\
\hline \hline
\end{tabular}

Note: Percentages are based on nine trials for subjects Yellow 23, Green 1076 and DV 312, and on six trials for Yellow 2, White 8174 and DV 753.

$\$$ - based on ten trials. ${ }^{*}$ - based on five trials. ${ }^{\wedge}$ - based on four trials.

Memory Test

As seen in Table 3, the overall tendency to visit items in the correct sequence was low

382 (final column "correct sequence"), with the highest success rate of $45 \%$ shown by the

383 youngest subject, Yellow 2. To analyse performance, the following chunks within the

384 sequence were considered: choosing sequence item 1 first, choosing item 2 directly after item

385 1, item 3 directly after 2, item 4 directly after 3 (if applicable), and choosing the final trained

386 item last. For the group experiencing up to TS3, the percentage of trials during which a subject

387 completed a chunk of one or two items in their correct order significantly decreased the later

388 the chunk appeared in the sequence (Friedman test: $\chi^{2}{ }_{3}=9.0, P=0.029$ ). Pairwise post-hoc

389 comparisons (Durbin-Conover) further confirmed that the percentage of choosing sequence

390 item 1 first was significantly higher than choosing later chunks (comparisons of " 1 chosen

391 first" to any other option: $P \leq 0.001$ ), and lowest for choosing sequence item 3 after item 2

392 and for avoiding the last item until the ultimate choice (comparison of " 2 chosen after 1" to

393 "3 chosen after 2 " and to " 3 chosen last": both $P<0.001$; comparison of " 3 chosen after 2" to

394 “3 chosen last": $P=1.0$ ). Similarly, performance was significantly affected by the position of 
395 the chunk within the sequence for the subjects experiencing TS4 (Friedman test: $\chi^{2}{ }_{4}=12.2, P$

$396=0.016$ ). The percentage of choosing sequence item 1 first was significantly higher than

397 choosing later chunks (comparisons of " 1 chosen first" to any other option: Durbin-Conover

398 tests: all $P \leq 0.016$ ). Pigeons also completed the chunk " 2 chosen after 1 " significantly more

399 often than " 3 chosen after 2" and " 4 chosen last" (Durbin-Conover tests: both $P \leq 0.036$; any

400 other comparisons: $P \geq 0.16$ ). Taken together, all pigeons were decreasingly likely to

401 complete a chunk the later it appeared within the sequence. This decrease was visible as early

402 as the second sequence item, as predominantly only the first item was visited correctly.

403

404

Table 3. Percentage of Memory Test trials (out of ten trials) during which a subject chose sequence item 1 before any other items (" 1 first"), chose sequence item 2 directly after item 1 (" $1 \rightarrow 2$ "), chose sequence item 3 directly after item 2 (" $2 \rightarrow 3$ "), chose sequence item 4 directly after item 3 (" $3 \rightarrow 4$ "; only for those pigeons that had experienced training stage 4), chose the final trained items as its last choice ("3/4 last"), and when a subject completed the entire sequence of either three or four trained items in its correct order (" $1 \rightarrow 2 \rightarrow 3(\rightarrow 4)$ "). Individuals are listed in order of the number of items they were trained on; those that only completed up to TS3 were not expected to visit sequence item 4.

\begin{tabular}{|c|c|c|c|c|c|c|}
\hline Subject & 1 first & $1 \rightarrow 2$ & $2 \rightarrow 3$ & $3 \rightarrow 4$ & 3/4 last & $1 \rightarrow 2 \rightarrow 3(\rightarrow 4)$ \\
\hline Yellow 23 & $80 \%$ & $40 \%$ & $30 \%$ & - & $30 \%$ & $30 \%$ \\
\hline Green 1076 & $70 \%$ & $30 \%$ & $10 \%$ & - & $10 \%$ & $10 \%$ \\
\hline DV 312 & $80 \%$ & $30 \%$ & $20 \%$ & - & $20 \%$ & $20 \%$ \\
\hline Average & $77 \%$ & $33 \%$ & $20 \%$ & - & $20 \%$ & $20 \%$ \\
\hline Yellow 2 & $100 \%^{\wedge}$ & $64 \%^{\wedge}$ & $45 \% \wedge$ & $73 \%^{\wedge}$ & $45 \%^{\wedge}$ & $45 \%{ }^{\wedge}$ \\
\hline White 8174 & $100 \%$ & $70 \%$ & $50 \%$ & $30 \%$ & $10 \%$ & $10 \%$ \\
\hline Orange 11 & $67 \% *$ & $33 \% *$ & $33 \% *$ & $33 \% *$ & $33 \% *$ & $33 \% *$ \\
\hline DV 573 & $82 \%^{\wedge}$ & $36 \%{ }^{\wedge}$ & $9 \%^{\wedge}$ & $18 \%^{\wedge}$ & $18 \%^{\wedge}$ & $0 \%^{\wedge}$ \\
\hline Average & $87 \%$ & $51 \%$ & $34 \%$ & $39 \%$ & $27 \%$ & $22 \%$ \\
\hline
\end{tabular}

${ }^{*}$ - based on three trials. ${ }^{\wedge}$ - based on 11 trials.

DISCUSSION 
411 pigeons suffered impairments when compared to their younger conspecifics, indicating some

412 interindividual variability that emerges with age. The cognitive processes underlying

413 sequence learning, however, appeared to be independent of age, as all pigeons performed in

414 a way that indicated a mental encoding of sequence order.

415 The training data provided insight into an individual's ability to remember sequences

416 of increasing length. Lists of one and two items were acquired with relative ease by the

417 pigeons regardless of age. However, as early as the three-item sequence, pronounced

418 differences in acquisition rates emerged between the three age groups. Young pigeons

419 learned the list within significantly fewer sessions than adult and aged pigeons, and half of

420 the adult and aged individuals were unable to reproduce the sequence sufficiently within the

421 session limit. No subject reached the learning criterion for the four-item sequence. Although

422 the criterion was deliberately set relatively high to avoid ceiling effects, it was expected that

423 at least the younger pigeons would be capable of reaching this standard, as the one-year-old

424 pigeons in Terrace's 5,6 experiment did achieve a comparable level of performance not only

425 for four-item lists but also for five-item lists. Considering that the current study employed an

426 open-field paradigm, whereas previous studies used computerised tasks, it is difficult to

427 pinpoint the cause of this discrepancy, which could range from visual aspects and differences

428 in the mental representation of 2D and 3D stimuli (cf. ${ }^{8-10}$ ) to motoric aspects when pigeons

429 have to move only their heads compared to their entire body from one stimulus to the next.

430 To acquire a measure of progress with the four-item list, we compared the highest

431 reached daily score (correctly completed trials out of the ten daily trials) after the pigeons

432 had received 30 of the 60 TS4 sessions. Although the highest scores were achieved by the

433 youngest age group, older subjects did not fall far behind, and all subjects that had reached

434 this training stage reached the threshold set for success in other related studies (i.e., $30 \%$

435 correct in a single session ${ }^{4}$ ). The lack of clear age differences in TS4 despite observable 
436 differences in TS3 suggests that age does not affect pigeons' memory performance uniformly.

437 As is the case for humans ${ }^{11}$, there appear to be well-aging individual pigeons that preserve a

438 high level of memory capacity (comparable to the performance of the least successful young

439 subjects) and less well-aging individuals that show noticeable declines in memory. During a

440 highly complex memory task such as the simultaneous-chaining task presented here, the

441 decline is already noticeable at a relatively early age.

442 We incorporated two tests, a Choice Test and a Memory Test, to assess pigeons' 443 encoding of sequential order and infer potential differences regarding the cognitive 444 mechanisms underlying sequence learning across the age groups. There was no indication in 445 the obtained data to suggest performance differed between young, adult and aged pigeons in 446 the two administered tests.

447 The Choice Test allowed for a comparison of the observed behaviour to previous 448 studies. When presented with a forced choice between two list items, pigeons chose the first 449 list item over items presented later in the sequence, and chose earlier items over the final list 450 item, but they showed no preference for earlier sequence items when the choice was between 451 two items presented in the middle of the list. Terrace 5 interpreted these findings as evidence 452 that pigeons chained responses to neighbouring items within a sequence, learning that a 453 response to item $n$ was only correct when preceded by a response to item $n-1$, but with no 454 further reasoning about sequential order among the items. He further asserted that the 455 special positions of the first and the last sequence items were encoded separately, resulting 456 in correctly ordered responses when the choices included one of these items, but disrupted 457 performance when the choice did not include the first or the last item. The results from our 458 Choice Test only replicate Terrace's findings in part - our pigeons also showed their weakest 459 choice performance during trials that included internal items of the sequence ( 2 vs 3 for those 460 subjects that had trained with four items). However, performance was just as impaired when 
461 the choice included the two final items ( 3 vs 4 , or 2 vs 3 for those subjects that had trained 462 with three items), an observation that is directly contrary to Terrace's argument of the final 463 item assuming a "special role". Indeed, reduced performance occurred primarily when the 464 choice was between sequentially neighbouring items, regardless of whether the item pair 465 occurred in the middle or at the end of the sequence. Item pairs that did not consist of 466 immediately neighbouring items within the sequence reliably led to good performance (2 vs 467 4), which was at the same level of accuracy as any choices including item 1 . The special role 468 of the first item also mentioned by Terrace persisted in the current study as well, however, 469 this was most likely due to overlearning. Taken together, these results suggest that, instead 470 of associatively chaining responses to neighbouring items, during the current experiment the 471 pigeons encoded the order of items to some degree, although it might have been more in 472 terms of items that appear "earlier" or "later" within the sequence than in terms of individual 473 ordinal positions. The salience of the last item, which was so prominent in Terrace's studies, 474 was not confirmed here. However, Terrace's pigeons were trained on five-item lists, which 475 might have posed an additional level of cognitive difficulty to his subjects, resulting in good 476 memory only of the first and last item but with little representation of the order of internal 477 items. Instead, as noted by Scarf and Colombo ${ }^{4}$, Terrace's subjects relied on the display of all 478 five list items together to be able to reproduce the order. With shorter lists of four or three 479 items, as used in this experiment, the cognitive load may have been reduced sufficiently to 480 allow an encoding of the relative position of items as appearing earlier or later within the list. 481 The Memory Test allowed a further assessment of whether the observed behaviour 482 supported the hypothesis proposed by Terrace ${ }^{5}$ regarding chaining of responses without an 483 inherent concept of order, or the more cognitively complex process proposed by Scarf and 484 Colombo ${ }^{4}$. The most obvious result of this test was that item pairs further down the list were 485 increasingly less likely to be chosen in their correct order. It is unlikely that this failure to 
adhere to the trained sequence stemmed from a lack of memory of the later items themselves

487 - although the subjects had received less training sessions with later sequence items overall,

488 all items were equally preferred over novel items in the pairwise-choice test. Instead, we

489 observed that subjects abandoned the sequence as soon as their visit to the first item resulted

490 in an absence of reinforcement. This observation is difficult to combine with Terrace's

491 account of chained responses, by which responding to one item automatically cues the

492 response to the next. As the pigeon had to complete two baseline trials correctly before

493 entering a Memory Test trial, it is unlikely that the sudden stop was due to a lack of motivation

494 or an inability to remember the sequence that the pigeon had completed successfully in the

495 immediately preceding trial. Although unexpected, the observed behaviour indicates that

496 pigeons had formed a concept of the goal of completing the sequence (to maximise reward)

497 and acted in a planned way to achieve it. The pigeons did not perceive each item as an

498 individual stimulus-outcome event, but indeed as part of a connected sequence. When the

499 expected reward was not encountered in sequence item 1 during the non-reinforced test

500 trials, this disruption was likely extrapolated to the entire sequence and the subjects changed

501 their behaviour from the formerly goal-directed completion of the sequence to an

502 unstructured search.

503 The combination of results of both tests makes it evident that the arguments put

504 forward by Terrace ${ }^{5}$ are not compatible with the behaviour shown by our pigeons. Instead

505 of acquiring simple response-response associations, the pigeons showed evidence of forming

506 a representation of the order of list items, although this representation might be less

507 sophisticated than proposed by Scarf and Colombo ${ }^{4}$, and instead limited to a concept of items

508 that appear earlier and later in the sequence. As Terrace pointed out correctly, the first item

509 takes a special role, likely due to excessive overtraining. Furthermore, although the absolute

510 length of the sequence that could effectively be memorised was reduced for some older 
511 pigeons, the ability to form a mental representation of order was preserved in all pigeons.

512 Regardless of age, pigeons were able to perform in a way that was consistent with the task 513 goal of maximising reward.

514 Thus, the current study showed that age can have a noticeable effect on the cognition

515 of pigeons. As previously shown for humans 12,13, aging does not impact all cognitive abilities

516 uniformly, nor are all individuals affected to the same degree. It is possible to identify

517 cognitive capacities that are highly susceptible to age for many species, such as memory, as

518 shown in this study. Our study further supports that aging is an individual process,

519 manifesting itself differently in "healthy-aging" or "poorly-aging" individuals. Although aging

520 research is currently still limited to a few species, it is evident that age plays a crucial role in

521 many aspects of animal cognition and behaviour. Identifying suitable paradigms, like the one

522 presented in this study, to investigate such age effects in a wide range of species is the first 523 step to closing the gap. 


\section{REFERENCES}

525 1. Alexander, G. E. et al. Characterizing cognitive aging in humans with links to animal

526 models. Front. Aging Neurosci. 4, 1-18 (2012).

527 2. Craik, F. I. M. \& Rose, N. S. Memory encoding and aging: A neurocognitive perspective.

$528 \quad$ Neurosci. Biobehav. Rev. 36, 1729-1739 (2012).

529 3. Gallagher, M. \& Rapp, P. R. The use of animal models to study the effects of aging on

$530 \quad$ cognition. Annu. Rev. Psychol. 48, 339-70 (1997).

531 4. Scarf, D. \& Colombo, M. Serial List Learning. in Encyclopedia of Animal Cognition and

532 Behavior (eds. Vonk, J. \& Shackelford, T.) 1-10 (Springer International Publishing,

533 2018). doi:10.1007/978-3-319-47829-6_1507-1.

$534 \quad 5 . \quad$ Terrace, H. S. The Comparative Psychology of Serially Organized Behavior. Comp.

535 Cogn. Behav. Rev. 5, 23-58 (2010).

536 6. Terrace, H. S. The simultaneous chain: A new approach to serial learning. Trends

537 Cogn. Sci. 9, 202-210 (2005).

538 7. jamovi project. jamovi (Version 1.2) [Computer Software]. Retrieved from

539 https://www.jamovi.org (2020).

540 8. Weisman, R. \& Spetch, M. Determining When Birds Perceive Correspondence

541 Between Pictures and Objects: A Critique. Comp. Cogn. Behav. Rev. 5, 117-131 (2010).

542 9. Bovet, D. \& Vauclair, J. Picture recognition in animals and humans. Behav. Brain Res.

$543 \quad 109,143-165(2000)$.

544 10. Fagot, J. Picture perception in animals. (London: Psychology Press Ltd, 2000).

545 11. Cabeza, R., Anderson, N. D., Locantore, J. K. \& McIntosh, A. R. Aging gracefully:

$546 \quad$ Compensatory brain activity in high-performing older adults. Neuroimage 17, 1394-

$547 \quad 1402$ (2002).

548 12. Murman, D. L. The Impact of Age on Cognition. Semin. Hear. 36, 111-121 (2015). 
549 13. Salthouse, T. A. Selective review of cognitive aging. J. Int. Neuropsychol. Soc. 16, 754$760(2010)$. 
552 We thank Harshita Kushwaha, Abdelrahman Elzayadi, Alizée Vernouillet and Kevin

553 Leonard for their valuable assistance during the development and execution of this task.

554 Research support was provided by a Research Manitoba Postgraduate Fellowship granted to

$555 \mathrm{CM}$, an Undergraduate Student Research Award from the National Science and Engineering

556 Council and an Undergraduate Student Research Award from the University of Manitoba

557 granted to PS, and a Natural Science and Engineering Research Council Discovery grant

558 (\#4944-2017) and Canada Research Chair fund to DMK.

\section{AUTHOR CONTRIBUTIONS}

562 CM designed the study, CM and PS created the material and conducted the

563 experiment, CM analysed and interpreted the data and wrote the manuscript, PS and DK

564 edited the manuscript. All authors approve the submitted version and agree to be personally

565 accountable for their own contributions and to ensure that questions related to the accuracy

566 or integrity of any part of the work, even ones in which the author was not personally

567 involved, are appropriately investigated, resolved, and the resolution documented in the

568 literature.

569

570

571 ADDITIONAL INFORMATION

572 The authors declare no competing interests. 
575 (in grey) were placed in a circular array in one half of the arena. The pigeon entered at the

576 opposite side of the arena (the approximate location is marked in red). The insert in the 577 broken-line box shows a 3D model of a feeder.

Figure 2. Examples of a trial in each memory training stage. The location of each sequence item (coloured feeder) changed between trials as to not systematically reinforce a certain location. The sequence shown here is for illustrative purposes.

Figure 3. Mean number of sessions required to reach completion criterion in each training stage (TS) for each age group. Individual subject data are plotted as point

583 values. Error bars represent standard errors.

Table 1. Percentage of trials (out of three trials) during which a subject chose the item that had appeared earlier in the sequence. Individuals are listed in order of the number of items they received during training; those that only completed up to TS3 were not presented with choices including sequence item 4 .

Table 2. Percentage of Choice Test trials during which a subject chose the known item

590 when the choice was between a known (sequence items 1, 2, 3, or 4, as applicable) and an 591 unknown item (sequence items 5, 6, or 4, as applicable), or between two unknown items. 592 Individuals are listed in order of the number of items they were trained on; those that only 593 completed up to TS3 were not presented with sequence item 4 as a known option.

594 Table 3. Percentage of Memory Test trials (out of ten trials) during which a subject 595 chose sequence item 1 before any other items (" 1 first"), chose sequence item 2 directly after 596 item 1 (“1 2 2"), chose sequence item 3 directly after item 2 (“2 2 3"), chose sequence item 4 597 directly after item 3 ("3 2 4"; only for those pigeons that had experienced training stage 4), 
598 chose the final trained items as its last choice ("3/4 last"), and when a subject completed the 599 entire sequence of either three or four trained items in its correct order (“1 2 2 目 3 (园 4)”).

600 Individuals are listed in order of the number of items they were trained on; those that only 601 completed up to TS3 were not expected to visit sequence item 4. 


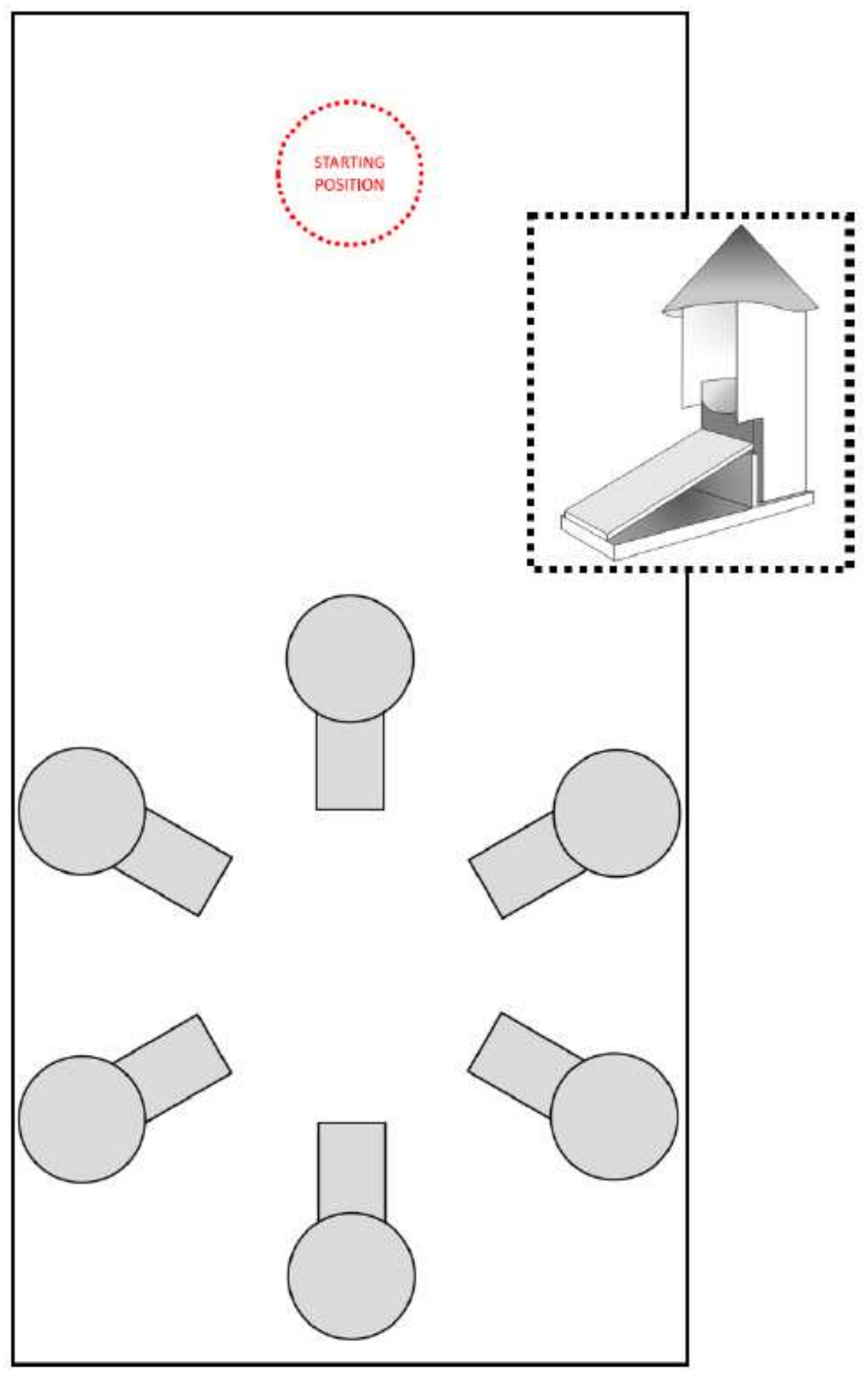

Figure 1

Top-down view of the inside of the experimental arena. The six feeders (in grey) were placed in a circular array in one half of the arena. The pigeon entered at the opposite side of the arena (the approximate location is marked in red). The insert in the broken-line box shows a 3D model of a feeder. 
a: Training Stage 1

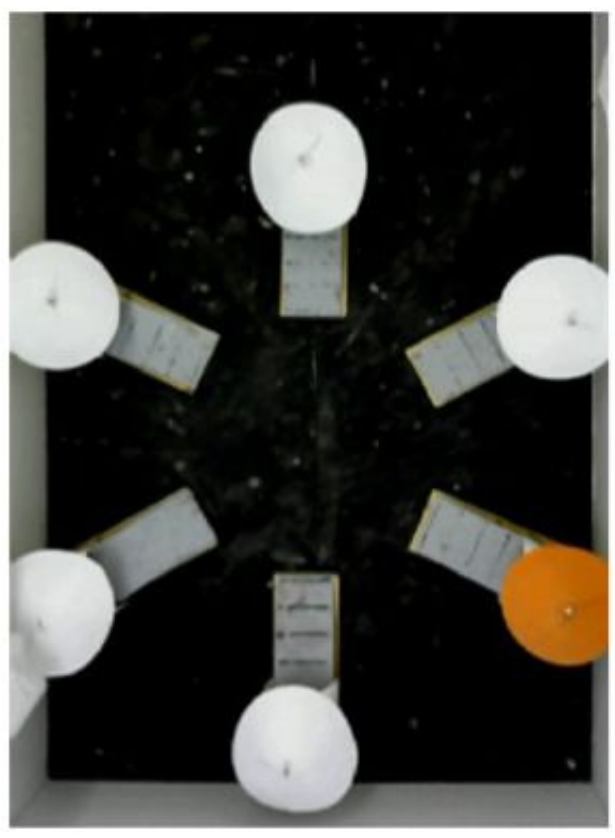

c: Training Stage 3

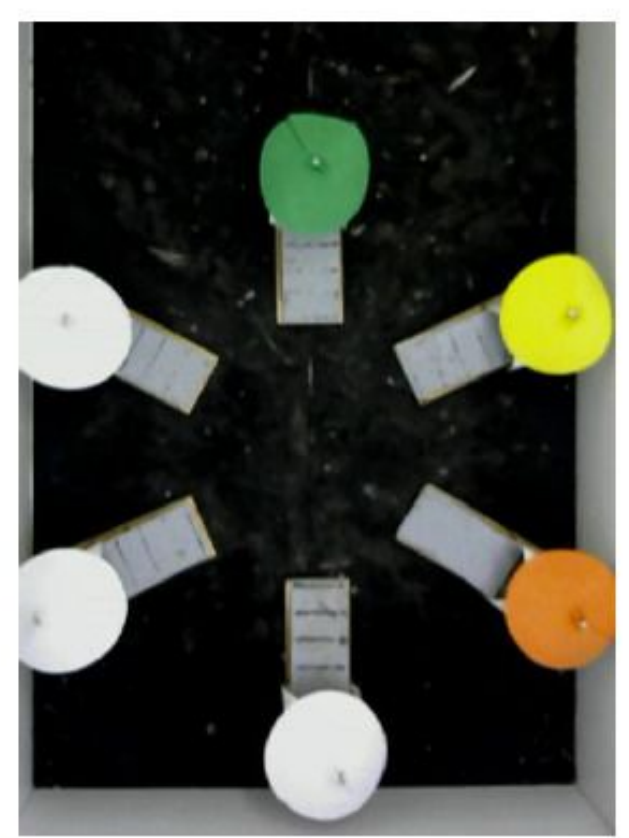

b: Training Stage 2

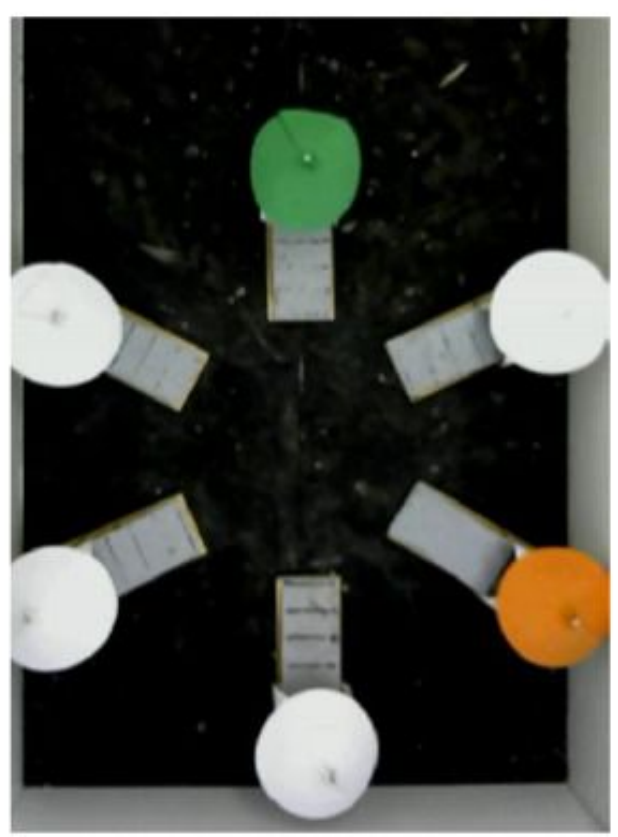

d: Training Stage 4

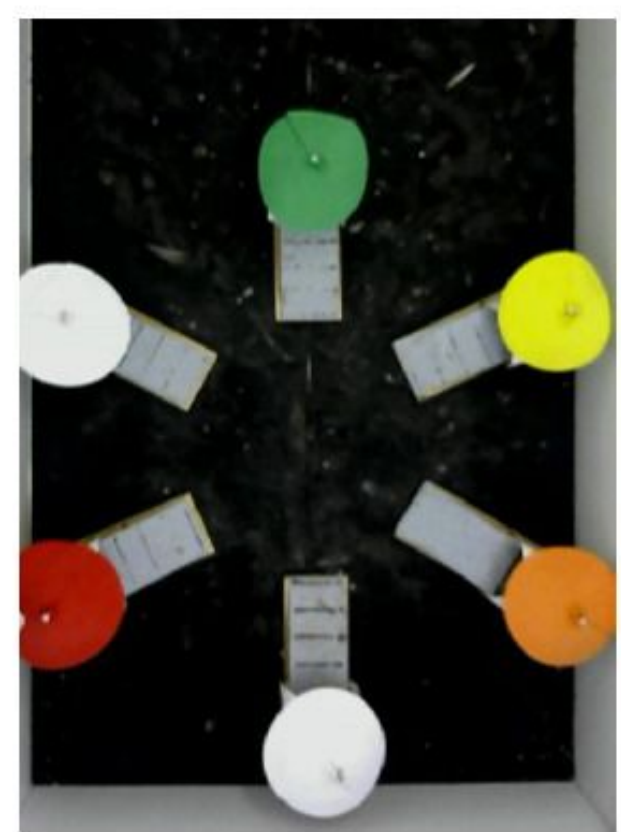

\section{Figure 2}

Examples of a trial in each memory training stage. The location of each sequence item (coloured feeder) changed between trials as to not systematically reinforce a certain location. The sequence shown here is for illustrative purposes. 


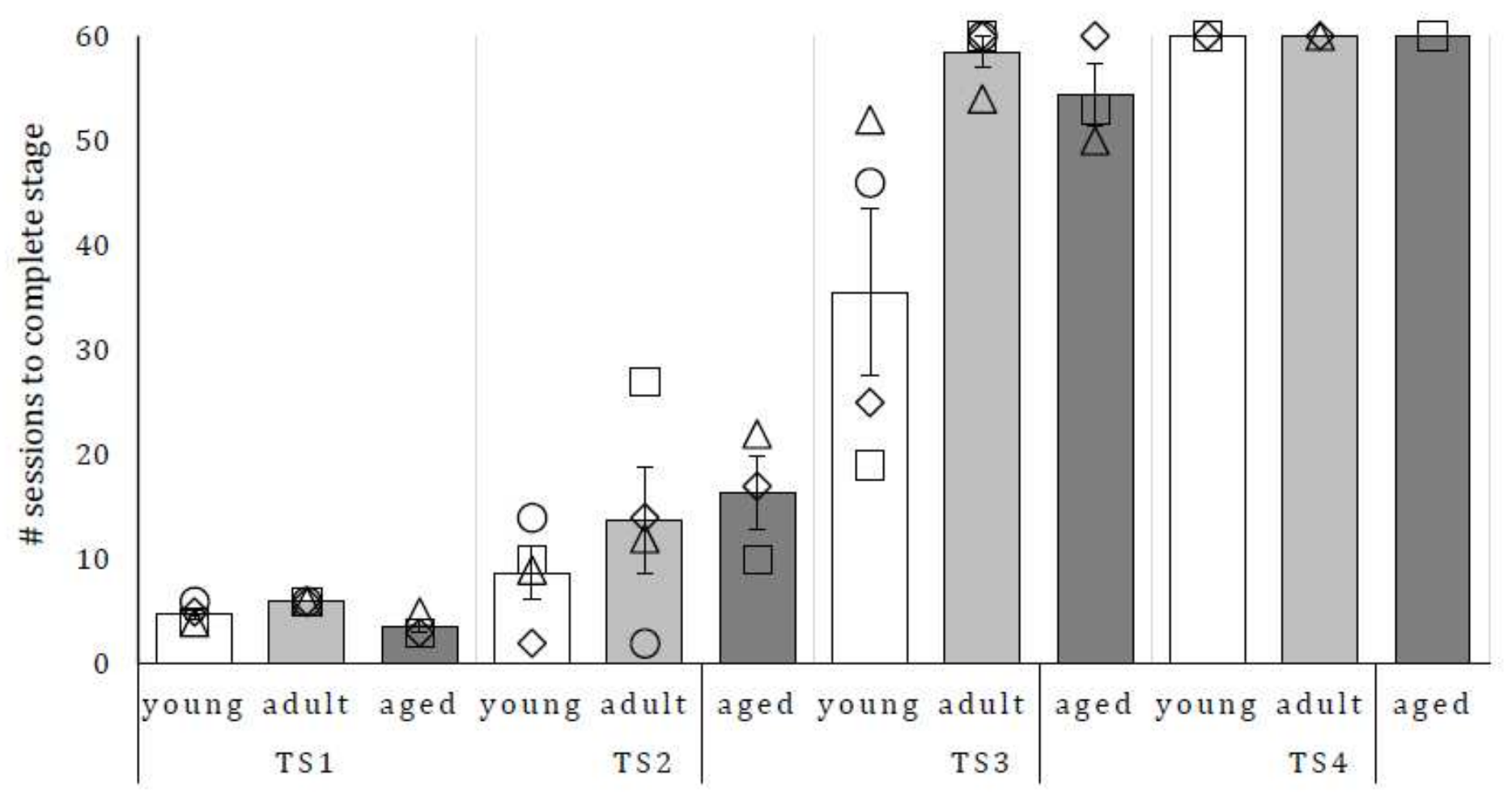

Figure 3

Mean number of sessions required to reach completion criterion in each training stage (TS) for each age group. Individual subject data are plotted as point values. Error bars represent standard errors. 